\title{
Inverse spectral problem of a class of fourth-order eigenparameter-dependent boundary value problems
}

\author{
Ji-jun $\mathrm{Ao}^{1 *}$ and Liang Zhang ${ }^{1}$
}

\section{"Correspondence: \\ george_ao78@sohu.com \\ ${ }^{1}$ College of Sciences, Inner \\ Mongolia University of Technology, \\ Hohhot, China}

\begin{abstract}
This paper deals with a class of inverse spectral problems of fourth-order boundary value problems with eigenparameter-dependent boundary conditions. Under the equivalent conditions of the problem and a certain type of matrix eigenvalue problem some coefficient functions are reconstructed from the given three sets of interlacing real numbers and several additional conditions. The key technique is the method of inverse matrix eigenvalue problems of a two-banded matrix.
\end{abstract}

MSC: Primary 34A55; 34B09; secondary $15 \mathrm{~A} 29$

Keywords: Inverse spectral problems; Inverse matrix eigenvalue problems; Fourth-order problems; Eigenparameter-dependent boundary conditions; Block pseudo-Jacobi matrix; Two-banded matrix

\section{Introduction}

This paper is a generalization of [26] and [5]. In [26], a class of inverse problems of SturmLiouville problems with eigenparameter-dependent boundary conditions has been considered by the authors. And in [5], the authors studied the inverse fourth-order boundary value problems with finite spectrum under separated boundary conditions. Since the higher-order boundary value problems with eigenparameter-dependent boundary conditions have their own theoretical and application background, we will investigate an inverse spectral problem of the fourth-order boundary value problems with eigenparameterdependent boundary conditions. The reader may find the historical and research background on boundary value problems with eigenparameter-dependent boundary conditions in $[20,26]$ and the references therein.

It is well known that the inverse spectral problems play an important role in many scientific fields which is motivated by recovering operators from their spectral data. Such problems have been widely studied by mathematicians, physicists, and engineers. Especially the inverse matrix eigenvalue problems and inverse Sturm-Liouville problems have been deeply studied in the last several decades [7, 8, 10, 11, 13, 22-25]. Such problems are always connected with the problems such as the vibrating systems [12], the classical moment problems [19], and quantum mechanics [18].

(c) The Author(s) 2020. This article is licensed under a Creative Commons Attribution 4.0 International License, which permits use, sharing, adaptation, distribution and reproduction in any medium or format, as long as you give appropriate credit to the original author(s) and the source, provide a link to the Creative Commons licence, and indicate if changes were made. The images or other third party material in this article are included in the article's Creative Commons licence, unless indicated otherwise in a credit line to the material. If material is not included in the article's Creative Commons licence and your intended use is not permitted by statutory regulation or exceeds the permitted use, you will need to obtain permission directly from the copyright holder. To view a copy of this licence, visit http://creativecommons.org/licenses/by/4.0/. 
In recent years, boundary value problems (BVPs) of Atkinson type which have a finite spectrum have been considered by some scholars $[1-4,6,14,15,17]$. These problems are connected with some physical problems such as frequencies of vibrating strings and diffusion operators [21]. Besides the finite spectrum results and matrix representations of these problems, the corresponding inverse spectral problems of such a type of problems have also been investigated most recently [5, 9, 16, 26, 27]. In 2012, Kong and Zettl considered the inverse Sturm-Liouville problems with finite spectrum of Atkinson type by using the so-called matrix representations of these problems [16]. In 2018, Cai and Zheng [9] generated the results in [16] and investigated a class of inverse Sturm-Liouville problems with discontinuous boundary conditions. The inverse spectral problems of Sturm-Liouville problems with eigenparameter- dependent boundary conditions and fourth-order boundary value problems were studied by Zhang and Ao in [26, 27] and [5], respectively. Since the fourth-order boundary value problems and the eigenparameter-dependent boundary value problems have their own significant background, we will discuss an inverse spectral problem on it.

Let us consider the fourth-order boundary value problem generated by the equation

$$
\left(p y^{\prime \prime}\right)^{\prime \prime}+q y=\lambda w y, \quad \text { on } I=[a, b],-\infty<a<b<+\infty,
$$

where the coefficients satisfy the condition

$$
r=1 / p, q, w \in L(I, \mathbb{R})
$$

here $L(I, \mathbb{R})$ denotes the real valued functions which are Lebesgue integrable on $I$, and the eigenparameter-dependent boundary conditions (BCs)

$$
A_{\lambda} Y(a)+B_{\lambda} Y(b)=0, \quad Y=\left[y, y^{\prime}, p y^{\prime \prime},\left(p y^{\prime \prime}\right)^{\prime}\right]^{T},
$$

where

$$
\begin{aligned}
A_{\lambda} & =\left[\begin{array}{cccc}
\lambda \alpha_{1}^{\prime}+\alpha_{1} & \lambda \alpha_{2}^{\prime}+\alpha_{2} & \lambda \alpha_{3}^{\prime}+\alpha_{3} & \lambda \alpha_{4}^{\prime}+\alpha_{4} \\
\lambda \beta_{1}^{\prime}+\beta_{1} & \lambda \beta_{2}^{\prime}+\beta_{2} & \lambda \beta_{3}^{\prime}+\beta_{3} & \lambda \beta_{4}^{\prime}+\beta_{4} \\
0 & 0 & 0 & 0 \\
0 & 0 & 0 & 0
\end{array}\right], \\
B_{\lambda} & =\left[\begin{array}{cccc}
0 & 0 & 0 & 0 \\
0 & 0 & 0 & 0 \\
\lambda \gamma_{1}^{\prime}+\gamma_{1} & \lambda \gamma_{2}^{\prime}+\gamma_{2} & \lambda \gamma_{3}^{\prime}+\gamma_{3} & \lambda \gamma_{4}^{\prime}+\gamma_{4} \\
\lambda \delta_{1}^{\prime}+\delta_{1} & \lambda \delta_{2}^{\prime}+\delta_{2} & \lambda \delta_{3}^{\prime}+\delta_{3} & \lambda \delta_{4}^{\prime}+\delta_{4}
\end{array}\right]
\end{aligned}
$$


with $\alpha_{i}, \alpha_{i}^{\prime}, \beta_{i}, \beta_{i}^{\prime}, \gamma_{i}, \gamma_{i}^{\prime}, \delta_{i}, \delta_{i}^{\prime} \in \mathbb{R}, i=1,2,3,4$, and satisfying

$$
\begin{array}{lll}
\operatorname{rank}\left(\begin{array}{llll}
\alpha_{1} & \alpha_{2} & \alpha_{3} & \alpha_{4} \\
\alpha_{1}^{\prime} & \alpha_{2}^{\prime} & \alpha_{3}^{\prime} & \alpha_{4}^{\prime}
\end{array}\right)=2, & \operatorname{rank}\left(\begin{array}{llll}
\beta_{1} & \beta_{2} & \beta_{3} & \beta_{4} \\
\beta_{1}^{\prime} & \beta_{2}^{\prime} & \beta_{3}^{\prime} & \beta_{4}^{\prime}
\end{array}\right)=2, \\
\operatorname{rank}\left(\begin{array}{llll}
\gamma_{1} & \gamma_{2} & \gamma_{3} & \gamma_{4} \\
\gamma_{1}^{\prime} & \gamma_{2}^{\prime} & \gamma_{3}^{\prime} & \gamma_{4}^{\prime}
\end{array}\right)=2, & \operatorname{rank}\left(\begin{array}{llll}
\delta_{1} & \delta_{2} & \delta_{3} & \delta_{4} \\
\delta_{1}^{\prime} & \delta_{2}^{\prime} & \delta_{3}^{\prime} & \delta_{4}^{\prime}
\end{array}\right)=2, \\
\operatorname{rank}\left(\begin{array}{llll}
\alpha_{1} & \alpha_{2} & \alpha_{3} & \alpha_{4} \\
\beta_{1} & \beta_{2} & \beta_{3} & \beta_{4}
\end{array}\right)=2, & \operatorname{rank}\left(\begin{array}{cccc}
\alpha_{1}^{\prime} & \alpha_{2}^{\prime} & \alpha_{3}^{\prime} & \alpha_{4}^{\prime} \\
\beta_{1}^{\prime} & \beta_{2}^{\prime} & \beta_{3}^{\prime} & \beta_{4}^{\prime}
\end{array}\right)=2, \\
\operatorname{rank}\left(\begin{array}{llll}
\gamma_{1} & \gamma_{2} & \gamma_{3} & \gamma_{4} \\
\delta_{1} & \delta_{2} & \delta_{3} & \delta_{4}
\end{array}\right)=2, & \operatorname{rank}\left(\begin{array}{llll}
\gamma_{1}^{\prime} & \gamma_{2}^{\prime} & \gamma_{3}^{\prime} & \gamma_{4}^{\prime} \\
\delta_{1}^{\prime} & \delta_{2}^{\prime} & \delta_{3}^{\prime} & \delta_{4}^{\prime}
\end{array}\right)=2 .
\end{array}
$$

Here $\lambda$ is the spectral parameter.

The fourth-order eigenparameter-dependent BVPs (1.1), (1.2) are of Atkinson type if for some positive integer $n \geq 1$, there exists a partition of the interval $[a, b]$

$$
a=a_{0}<b_{0}<a_{1}<b_{1}<\cdots<a_{n}<b_{n}=b,
$$

such that

$$
\begin{aligned}
& r(t)=\frac{1}{p(t)}=0 \quad \text { on }\left[a_{i}, b_{i}\right), i=0,1, \ldots, n, \quad \int_{b_{i-1}}^{a_{i}} r(t) d t \neq 0, \\
& \int_{b_{i-1}}^{a_{i}} r(t) t d t \neq 0, \quad \int_{b_{i-1}}^{a_{i}} r(t) t^{2} d t \neq 0, \quad i=1,2, \ldots, n ; \\
& q(t)=0 \quad \text { on }\left[b_{i-1}, a_{i}\right), i=1,2, \ldots, n,
\end{aligned}
$$

and

$$
\begin{gathered}
w(t)=0 \quad \text { on }\left[b_{i-1}, a_{i}\right), i=1,2, \ldots, n, \quad \int_{a_{i}}^{b_{i}} w(t) d t>0, \\
\int_{a_{i}}^{b_{i}} w(t) t d t>0, \quad \int_{a_{i}}^{b_{i}} w(t) t^{2} d t>0, \quad i=0,1, \ldots, n,
\end{gathered}
$$

and BC (1.2) satisfies the conditions (1.3).

To present our discussions in this paper we need to introduce the following notations. Let

$$
\begin{array}{lll}
r_{i}=\int_{b_{i-1}}^{a_{i}} r(t) d t, & \hat{r}_{i}=\int_{b_{i-1}}^{a_{i}} r(t) t d t, & \check{r}_{i}=\int_{b_{i-1}}^{a_{i}} r(t) t^{2} d t, \quad i=1,2, \ldots, n, \\
q_{i}=\int_{a_{i}}^{b_{i}} q(t) d t, & \hat{q}_{i}=\int_{a_{i}}^{b_{i}} q(t) t d t, & \check{q}_{i}=\int_{a_{i}}^{b_{i}} q(t) t^{2} d t, \quad i=0,1, \ldots, n, \\
w_{i}=\int_{a_{i}}^{b_{i}} w(t) d t, & \hat{w}_{i}=\int_{a_{i}}^{b_{i}} w(t) t d t, & \check{w}_{i}=\int_{a_{i}}^{b_{i}} w(t) t^{2} d t, \quad i=0,1, \ldots, n,
\end{array}
$$

and assume that

$$
\theta_{i}=\hat{r}_{i}^{2}-r_{i} \check{r}_{i} \neq 0, \quad i=1,2, \ldots, n .
$$


For a given equation (1.1) with coefficients satisfying (1.5)-(1.7), let us set

$$
\begin{array}{lll}
\vec{\alpha}=\left(\alpha_{1}, \alpha_{2}, \alpha_{3}, \alpha_{4}\right), & \overrightarrow{\alpha^{\prime}}=\left(\alpha_{1}^{\prime}, \alpha_{2}^{\prime}, \alpha_{3}^{\prime}, \alpha_{4}^{\prime}\right), & \vec{\beta}=\left(\beta_{1}, \beta_{2}, \beta_{3}, \beta_{4}\right), \\
\overrightarrow{\beta^{\prime}}=\left(\beta_{1}^{\prime}, \beta_{2}^{\prime}, \beta_{3}^{\prime}, \beta_{4}^{\prime}\right), & \vec{\gamma}=\left(\gamma_{1}, \gamma_{2}, \gamma_{3}, \gamma_{4}\right), & \overrightarrow{\gamma^{\prime}}=\left(\gamma_{1}^{\prime}, \gamma_{2}^{\prime}, \gamma_{3}^{\prime}, \gamma_{4}^{\prime}\right), \\
\vec{\delta}=\left(\delta_{1}, \delta_{2}, \delta_{3}, \delta_{4}\right), & \overrightarrow{\delta^{\prime}}=\left(\delta_{1}^{\prime}, \delta_{2}^{\prime}, \delta_{3}^{\prime}, \delta_{4}^{\prime}\right), & \overrightarrow{0}=(0,0,0,0) .
\end{array}
$$

Then we let $\sigma\left(\vec{\alpha}, \overrightarrow{\alpha^{\prime}}, \vec{\beta}, \overrightarrow{\beta^{\prime}}, \vec{\gamma}, \overrightarrow{\gamma^{\prime}}, \vec{\delta}, \vec{\delta}^{\prime}\right)$ be the spectrum of the fourth-order eigenparameterdependent BVPs (1.1), (1.2).

Assume $k \in \mathbb{N}^{+}$such that $k>2$, and let $\mathbb{M}_{2 k}$ be the set of $2 k \times 2 k$ matrices over the reals. For any $Y \in \mathbb{M}_{2 k}$, we denote by $\sigma(Y)$ the set of eigenvalues of $Y$. Furthermore, let $Y_{1}$ be the principal submatrix obtained from $Y$ by removing its first row and column, and $Y^{1}$ its submatrix obtained from $Y$ by removing the last row and column. Let $Y_{12}$ be the principal submatrix obtained from $Y$ by removing its first two rows and first two columns, and $Y^{12}$ its submatrix obtained from $Y$ by removing the last two rows and the last two columns. For any $2 \times 2$ matrix $F$, we denote by $Y_{12}[F]$ the matrix of $Y_{12}$ plus $F$ in the $(1,1),(1,2),(2,1),(2,2)$ entries. We denote by $Y^{12}[F]$ the matrix of $Y^{12}$ plus $F$ in the $(2 k-$ $3,2 k-3),(2 k-3,2 k-2),(2 k-2,2 k-3),(2 k-2,2 k-2)$ entries.

For any $Y, Z \in \mathbb{M}_{2 k}$, we say that $\lambda^{*}$ is an eigenvalue of the matrix-pair $(Y, Z)$ if there exists a nontrivial vector $u \in \mathbb{R}^{2 k}$ such that $\left(Y-\lambda^{*} Z\right) u=0$. We denote by $\sigma(Y, Z)$ the set of eigenvalues of $(Y, Z)$. Clearly, $\lambda^{*} \in \sigma(Y)$ if and only if $\lambda^{*} \in \sigma\left(Y, I_{2 k}\right)$, where $I_{2 k}$ is the identity matrix in $\mathbb{M}_{2 k}$.

\section{Equivalent matrix representations of fourth-order eigenparameter-dependent BVPs}

To illustrate our main result on inverse fourth-order eigenparameter-dependent BVPs, we will show the following lemmas, which have been given in [1].

Lemma 2.1 ([1]) Let $x_{1}, x_{2} \in \mathbb{R}$ and $x_{1} \neq x_{2}$. Then, for any $\eta_{0}, \eta_{1}, \eta_{2} \in \mathbb{R}$, there exists $a$ unique polynomial function $P(t)=\tau_{2} t^{2}+\tau_{1} t+\tau_{0}$, such that

$$
\int_{x_{1}}^{x_{2}} P(t) d t=\eta_{0}, \quad \int_{x_{1}}^{x_{2}} P(t) t d t=\eta_{1}, \quad \int_{x_{1}}^{x_{2}} P(t) t^{2} d t=\eta_{2} .
$$

Denote the polynomial constructed in Lemma 2.1 by $\chi\left(x_{1}, x_{2}, \eta_{0}, \eta_{1}, \eta_{2}\right)$ and define the piecewise polynomial functions $\bar{r}(t)=\frac{1}{\bar{p}(t)}, \bar{q}(t)$ and $\bar{w}(t)$ on $[a, b]$ by

$$
\begin{aligned}
& \bar{r}(t)=\frac{1}{\bar{p}(t)}= \begin{cases}\chi\left(b_{i-1}, a_{i}, r_{i}, \hat{r}_{i}, \check{r}_{i}\right), & t \in\left[b_{i-1}, a_{i}\right), i=1,2, \ldots, n ; \\
0, & t \in\left[a_{i}, b_{i}\right), i=0,1, \ldots, n ;\end{cases} \\
& \bar{q}(t)= \begin{cases}\chi\left(a_{i}, b_{i}, q_{i}, \hat{q}_{i}, \check{q}_{i}\right), & t \in\left[a_{i}, b_{i}\right), i=0,1, \ldots, n ; \\
0, & t \in\left[b_{i-1}, a_{i}\right), i=1,2, \ldots, n ;\end{cases} \\
& \bar{w}(t)= \begin{cases}\chi\left(a_{i}, b_{i}, w_{i}, \hat{w}_{i}, \check{w}_{i}\right), & t \in\left[a_{i}, b_{i}\right), i=0,1, \ldots, n ; \\
0, & t \in\left[b_{i-1}, a_{i}\right), i=1,2, \ldots, n .\end{cases}
\end{aligned}
$$

Then we can state the following lemma. 
Lemma $2.2([1])$ Assume $r(t), q(t), w(t) \in L(I, \mathbb{R})$ satisfy (1.5)-(1.7). Then the fourth-order eigenparameter-dependent BVPs (1.1), (1.2) have exactly the same eigenvalues as the fourth-order eigenparameter-dependent BVPs consisting of the equation with piecewise polynomial functions

$$
\left(\bar{p} y^{\prime \prime}\right)^{\prime \prime}+\bar{q} y=\lambda \bar{w} y, \quad \text { on } I=[a, b]
$$

and the same $B C(1.2)$.

It can be inferred from Lemma 2.2, for a fixed BC (1.2) and a given partition of the interval $[a, b]$, there is a family of fourth-order eigenparameter-dependent BVPs of Atkinson type which has exactly the same eigenvalues as fourth-order eigenparameterdependent BVP (2.2), (1.2). Such a family is called the equivalent family of the fourthorder eigenparameter-dependent BVPs (2.2), (1.2). It is clear that, for an equivalent family of the fourth-order eigenparameter-dependent BVPs (2.2), (1.2), the parameters $r_{i}, \hat{r}_{i}, \check{r}_{i}$ and $q_{i}, \hat{q}_{i}, \check{q}_{i}, w_{i}, \hat{w}_{i}, \check{w}_{i}$ defined by (1.8) are all the same.

Lemma 2.3 ([1]) Consider the $B C(1.2)$ with $\alpha_{i}, \alpha_{i}^{\prime}, \beta_{i}, \beta_{i}^{\prime}, \gamma_{i}, \gamma_{i}^{\prime}, \delta_{i}, \delta_{i}^{\prime} \in \mathbb{R}, i=1,2,3,4$, satisfying the conditions (1.3). Define an $(n+3) \times(n+3)$ block tridiagonal matrix

$$
\mathbf{P}=\left[\begin{array}{ccccccc}
B_{1} & B_{2} & & & & & \\
E_{2} & -P_{1} & P_{1} & & & & \\
& P_{1} & -P_{1}-P_{2} & P_{2} & & & \\
& & \cdots & \ldots & \ldots & & \\
& & & P_{n-1} & -P_{n-1}-P_{n} & P_{n} & \\
& & & & P_{n} & -P_{n} & -E_{2} \\
& & & & & B_{3} & B_{4}
\end{array}\right],
$$

a block diagonal matrix

$$
\mathbf{Q}=\operatorname{diag}\left(O, Q_{0}, Q_{1}, \ldots, Q_{n}, O\right)
$$

and an 'almost' block diagonal matrix

$$
\mathbf{W}=\left[\begin{array}{ccccccc}
-B_{1}^{\prime} & -B_{2}^{\prime} & & & & & \\
& W_{0} & & & & & \\
& & W_{1} & & & & \\
& & & \ldots & & & \\
& & & & W_{n-1} & & \\
& & & & & W_{n} & \\
& & & & & -B_{3}^{\prime} & -B_{4}^{\prime}
\end{array}\right] \text {, }
$$

where $E_{2}, O, P_{j}, j=1, \ldots, n$ and $Q_{j}, W_{j}, j=0,1, \ldots, n$, are $2 \times 2$ matrices with the rule

$$
E_{2}=\left[\begin{array}{cc}
0 & -1 \\
1 & 0
\end{array}\right], \quad O=\left[\begin{array}{cc}
0 & 0 \\
0 & 0
\end{array}\right], \quad P_{j}=\left[\begin{array}{cc}
\frac{r_{j}}{\theta_{j}} & \frac{\hat{r}_{j}}{\theta_{j}} \\
\frac{r_{j}}{\theta_{j}} & \frac{r_{j}}{\theta_{j}}
\end{array}\right], \quad j=1,2,3, \ldots, n,
$$




$$
Q_{j}=\left[\begin{array}{cc}
q_{j} & \hat{q}_{j} \\
\hat{q}_{j} & \check{q}_{j}
\end{array}\right], \quad W_{j}=\left[\begin{array}{cc}
w_{j} & \hat{w}_{j} \\
\hat{w}_{j} & \check{w}_{j}
\end{array}\right], \quad j=0,1, \ldots, n,
$$

and $B_{i}, B_{i}^{\prime}, i=1,2,3,4$, are $2 \times 2$ matrices with the rule

$$
\begin{array}{rlr}
B_{1}=\left[\begin{array}{ll}
\alpha_{3} & a \alpha_{3}+\alpha_{4} \\
\beta_{3} & a \beta_{3}+\beta_{4}
\end{array}\right], & B_{2}=\left[\begin{array}{ll}
\alpha_{1} & a \alpha_{1}+\alpha_{2} \\
\beta_{1} & a \beta_{1}+\beta_{2}
\end{array}\right], & B_{3}=\left[\begin{array}{ll}
\gamma_{3} & b \gamma_{3}+\gamma_{4} \\
\delta_{3} & b \delta_{3}+\delta_{4}
\end{array}\right], \\
B_{4}=\left[\begin{array}{ll}
\gamma_{1} & b \gamma_{1}+\gamma_{2} \\
\delta_{1} & b \delta_{1}+\delta_{2}
\end{array}\right], & B_{1}^{\prime}=\left[\begin{array}{ll}
\alpha_{3}^{\prime} & a \alpha_{3}^{\prime}+\alpha_{4}^{\prime} \\
\beta_{3}^{\prime} & a \beta_{3}^{\prime}+\beta_{4}^{\prime}
\end{array}\right], & B_{2}^{\prime}=\left[\begin{array}{ll}
\alpha_{1}^{\prime} & a \alpha_{1}^{\prime}+\alpha_{2}^{\prime} \\
\beta_{1}^{\prime} & a \beta_{1}^{\prime}+\beta_{2}^{\prime}
\end{array}\right], \\
B_{3}^{\prime}=\left[\begin{array}{ll}
\gamma_{3}^{\prime} & b \gamma_{3}^{\prime}+\gamma_{4}^{\prime} \\
\delta_{3}^{\prime} & b \delta_{3}^{\prime}+\delta_{4}^{\prime}
\end{array}\right], & B_{4}^{\prime}=\left[\begin{array}{ll}
\gamma_{1}^{\prime} & b \gamma_{1}^{\prime}+\gamma_{2}^{\prime} \\
\delta_{1}^{\prime} & b \delta_{1}^{\prime}+\delta_{2}^{\prime}
\end{array}\right] . &
\end{array}
$$

Then we have the same eigenvalues of the fourth-order eigenparameter-dependent BVPS (1.1), (1.2) and the matrix-pair $(\mathbf{P}+\mathbf{Q}, \mathbf{W})$, i.e.

$$
\sigma\left(\vec{\alpha}, \overrightarrow{\alpha^{\prime}}, \vec{\beta}, \overrightarrow{\beta^{\prime}}, \vec{\gamma}, \overrightarrow{\gamma^{\prime}}, \vec{\delta}, \vec{\delta}^{\prime}\right)=\sigma(\mathbf{P}+\mathbf{Q}, \mathbf{W})
$$

Lemma 2.4 Consider the $B C(1.2)$ with $\alpha_{i}^{\prime}, \beta_{i}^{\prime}, \gamma_{i}, \gamma_{i}^{\prime}, \delta_{i}, \delta_{i}^{\prime} \in \mathbb{R}, i=1,2,3,4$, satisfying the conditions (1.3). Define an $(n+2) \times(n+2)$ block tridiagonal matrix

$$
\begin{aligned}
& \mathbf{P}_{12}\left[-E_{2}\left(B_{1}^{\prime}\right)^{-1} B_{2}^{\prime}\right] \\
& =\left[\begin{array}{cccccc}
-P_{1}-E_{2}\left(B_{1}^{\prime}\right)^{-1} B_{2}^{\prime} & P_{1} & & & & \\
P_{1} & -P_{1}-P_{2} & P_{2} & & & \\
& \cdots & \cdots & \cdots & & \\
& & P_{n-1} & -P_{n-1}-P_{n} & P_{n} & \\
& & & P_{n} & -P_{n} & -E_{2} \\
& & & & B_{3} & B_{4}
\end{array}\right],
\end{aligned}
$$

a block diagonal matrix

$$
\mathbf{Q}_{12}=\operatorname{diag}\left(Q_{0}, Q_{1}, \ldots, Q_{n}, O\right),
$$

and an 'almost' block diagonal matrix

$$
\mathbf{W}_{12}=\left[\begin{array}{llllll}
W_{0} & & & & & \\
& W_{1} & & & & \\
& & \ldots & & & \\
& & & W_{n-1} & & \\
& & & & W_{n} & \\
& & & -B_{3}^{\prime} & -B_{4}^{\prime}
\end{array}\right] \text {, }
$$

where $E_{2}, O, P_{j}, j=1, \ldots, n, Q_{j}, W_{j}, j=0,1, \ldots, n$, and $B_{i}, i=3,4, B_{i}^{\prime}, i=1,2,3,4$ are $2 \times 2$ matrices as given in Lemma 2.3.

Then $\sigma\left(\overrightarrow{0}, \vec{\alpha}^{\prime}, \overrightarrow{0}, \vec{\beta}^{\prime}, \vec{\gamma}, \overrightarrow{\gamma^{\prime}}, \vec{\delta}, \vec{\delta}^{\prime}\right)=\sigma\left(\mathbf{P}_{12}+\mathbf{Q}_{12}, \mathbf{W}_{12}\right)$.

Proof The proof is similar to the proof of Lemma 2.3, hence it is omitted here. 
Lemma 2.5 Consider the $B C$ (1.2) with $\alpha_{i}, \alpha_{i}^{\prime}, \beta_{i}, \beta_{i}^{\prime}, \gamma_{i}^{\prime}, \delta_{i}^{\prime} \in \mathbb{R}, i=1,2,3,4$, satisfying the conditions (1.3). Define an $(n+2) \times(n+2)$ block tridiagonal matrix

$$
\begin{aligned}
\mathbf{P}^{12} & {\left[E_{2}\left(B_{4}^{\prime}\right)^{-1} B_{3}^{\prime}\right] } \\
& =\left[\begin{array}{cccccc}
B_{1} & B_{2} & & & & \\
E_{2} & -P_{1} & P_{1} & & & \\
& P_{1} & -P_{1}-P_{2} & P_{2} & & \\
& & \ldots & \ldots & \ldots & \\
& & & P_{n-1} & -P_{n-1}-P_{n} & P_{n} \\
& & & & P_{n} & -P_{n}+E_{2}\left(B_{4}^{\prime}\right)^{-1} B_{3}^{\prime}
\end{array}\right],
\end{aligned}
$$

a block diagonal matrix

$$
\mathbf{Q}^{12}=\operatorname{diag}\left(O, Q_{0}, Q_{1}, \ldots, Q_{n}\right)
$$

and an 'almost' block diagonal matrix

$$
\mathbf{W}^{12}=\left[\begin{array}{cccccc}
-B_{1}^{\prime} & -B_{2}^{\prime} & & & & \\
& W_{0} & & & & \\
& & W_{1} & & & \\
& & & \ldots & & \\
& & & & W_{n-1} & \\
& & & & & W_{n}
\end{array}\right] \text {, }
$$

where $E_{2}, O, P_{j}, j=1, \ldots, n, Q_{j}, W_{j}, j=0,1, \ldots, n$, and $B_{i}, i=1,2 ; B_{i}^{\prime}, i=1,2,3,4$ are $2 \times 2$ matrices as given in Lemma 2.3.

Then $\sigma\left(\vec{\alpha}, \overrightarrow{\alpha^{\prime}}, \vec{\beta}, \overrightarrow{\beta^{\prime}}, \overrightarrow{0}, \overrightarrow{\gamma^{\prime}}, \overrightarrow{0}, \vec{\delta}^{\prime}\right)=\sigma\left(\mathbf{P}^{12}+\mathbf{Q}^{12}, \mathbf{W}^{12}\right)$.

Proof The proof is similar to the proof of Lemma 2.3, hence it is omitted here.

Remark 2.1 In Lemmas 2.3, 2.4, 2.5, it is easy to conclude that

$$
(\mathbf{P}+\mathbf{Q})_{12}=\mathbf{P}_{12}+\mathbf{Q}_{12}, \quad(\mathbf{P}+\mathbf{Q})^{12}=\mathbf{P}^{12}+\mathbf{Q}^{12}
$$

\section{Related inverse matrix eigenvalue problems}

We first introduce the following matrices in $\mathbb{M}_{2 k}$ :

$$
\left[\begin{array}{cccccc}
\mathcal{C}_{1} & \mathcal{D}_{1} & & & & \\
\mathcal{D}_{1}^{T} & \mathcal{C}_{2} & \mathcal{D}_{2} & & & \\
& \ldots & \ldots & \ldots & & \\
& & & \mathcal{D}_{k-2}^{T} & \mathcal{C}_{k-1} & \mathcal{D}_{k-1} \\
& & & & \mathcal{D}_{k-1}^{T} & \mathcal{C}_{k}
\end{array}\right],
$$

where $\mathcal{C}_{i}, i=1, \ldots, k$ and $\mathcal{D}_{i}, i=1, \ldots, k-1$ are $2 \times 2$ matrices, $T$ denotes the transpose.

Definition 3.1 ([22]) A matrix $\mathbf{J}_{b} \in \mathbb{M}_{2 k}$ in the form of (3.1) is called a two-banded matrix if $\mathcal{C}_{i}=\left[\begin{array}{c}\grave{c}_{i} \bar{c}_{i} \\ \grave{c}_{i} \bar{c}_{i}\end{array}\right], \grave{c}_{i}, \bar{c}_{i}, \bar{c}_{i} \neq 0, i=1, \ldots, k ; \mathcal{D}_{i}=\left[\begin{array}{c}\grave{d}_{i} \\ \grave{d}_{i} \\ \grave{d}_{i}\end{array}\right], \grave{d}_{i}, \hat{d}_{i}, \bar{d}_{i} \neq 0, i=1, \ldots, k-1$. 
Lemma 3.1 ([22]) Let $\left\{\lambda_{i}: i=1, \ldots, 2 k\right\},\left\{\mu_{i}: i=1, \ldots, 2 k-1\right\}$ and $\left\{\xi_{i}: i=1, \ldots, 2 k-2\right\}$ be three sets of real numbers satisfying the interlacing property

$$
\begin{aligned}
& \lambda_{1} \leq \mu_{1} \leq \lambda_{2} \leq \mu_{2} \leq \cdots \leq \lambda_{2 k-1} \leq \mu_{2 k-1} \leq \lambda_{2 k} \\
& \mu_{1} \leq \xi_{1} \leq \mu_{2} \leq \xi_{2} \leq \cdots \leq \mu_{2 k-2} \leq \xi_{2 k-2} \leq \mu_{2 k-1} .
\end{aligned}
$$

Then there exists a two-banded matrix $\mathbf{J}_{b} \in \mathbb{M}_{2 k}$ such that

$$
\begin{aligned}
& \sigma\left(\mathbf{J}_{b}\right)=\left\{\lambda_{i}: i=1, \ldots, 2 k\right\}, \quad \sigma\left(\left(\mathbf{J}_{b}\right)_{1}\right)=\left\{\mu_{i}: i=1, \ldots, 2 k-1\right\}, \\
& \sigma\left(\left(\mathbf{J}_{b}\right)_{12}\right)=\left\{\xi_{i}: i=1, \ldots, 2 k-2\right\} .
\end{aligned}
$$

Next, we consider the block matrices in $\mathbb{M}_{2 k}$ of the form

$$
\left[\begin{array}{cccccc}
C_{1} & D_{1} & & & & \\
D_{1}^{\prime} & C_{2} & D_{2} & & & \\
& \ldots & \cdots & \ldots & & \\
& & & D_{k-2}^{T} & C_{k-1} & D_{k-1}^{\prime} \\
& & & & D_{k-1} & C_{k}
\end{array}\right],
$$

which is block symmetric except for the $D_{1}, D_{1}^{\prime}, D_{k-1}, D_{k-1}^{\prime}$ entries.

Definition 3.2 A matrix $\mathbf{M} \in \mathbb{M}_{2 k}$ in the form of (3.3) is called a $2 \times 2$ block pseudo-Jacobi matrix if $C_{i}=\left[\begin{array}{c}c_{i} \\ \hat{c}_{i} \\ \hat{c}_{i} c_{i}\end{array}\right], c_{i}, \hat{c}_{i}, \check{c}_{i} \neq 0, i=2, \ldots, k-1 ; D_{i}=\left[\begin{array}{c}d_{i} \hat{d}_{i} \\ \hat{d}_{i} \\ \breve{d}_{i}\end{array}\right], d_{i}, \hat{d}_{i}, \breve{d}_{i} \neq 0, i=2, \ldots, k-2$, and $C_{1}=\left[\begin{array}{ll}c_{11}^{(1)} & c_{12}^{(1)} \\ c_{21}^{(1)} & c_{22}^{(1)}\end{array}\right], D_{1}=\left[\begin{array}{ll}d_{11}^{(1)} & d_{12}^{(1)} \\ d_{21}^{(1)} & d_{22}^{(1)}\end{array}\right], D_{1}^{\prime}=\left[\begin{array}{ll}d_{11}^{(1)^{\prime}} & d_{12}^{(1)^{\prime}} \\ d_{21}^{(1)^{\prime}} & d_{22}^{(1)^{\prime}}\end{array}\right], D_{k-1}=\left[\begin{array}{lll}d_{11}^{(k-1)} & d_{12}^{(k-1)} \\ d_{21}^{(k-1)} & d_{22}^{(k-1)}\end{array}\right], D_{k-1}^{\prime}=\left[\begin{array}{lll}d_{11}^{(k-1)^{\prime}} & d_{12}^{(k-1)^{\prime}} \\ d_{21}^{(k-1)^{\prime}} & d_{22}^{(k-1)^{\prime}}\end{array}\right]$, $C_{k}=\left[\begin{array}{cc}c_{11}^{(k)} & c_{12}^{(k)} \\ c_{21}^{(k)} & c_{22}^{(k)}\end{array}\right]$ with each $D_{i}, i=1, \ldots, k-1$, and $C_{1}, C_{k}, D_{1}^{\prime}, D_{k-1}^{\prime}$ are $2 \times 2$ nonsingular matrices.

From Lemma 3.1, we can deduce the following theorem which includes the key technique to solve our main result.

Theorem 3.1 Let $\left\{\lambda_{i}: i=1, \ldots, 2 k\right\},\left\{\mu_{i}: i=1, \ldots, 2 k-1\right\}$ and $\left\{\xi_{i}: i=1, \ldots, 2 k-2\right\}$ be three sets of real numbers satisfying the interlacing property (3.2).

If

$$
\mathbf{W}=\left[\begin{array}{ccccccc}
-B_{1}^{\prime} & -B_{2}^{\prime} & & & & & \\
& W_{0} & & & & & \\
& & W_{1} & & & & \\
& & & \ldots & & & \\
& & & & W_{k-4} & & \\
& & & & & W_{k-3} & \\
& & & & & -B_{3}^{\prime} & -B_{4}^{\prime}
\end{array}\right]
$$

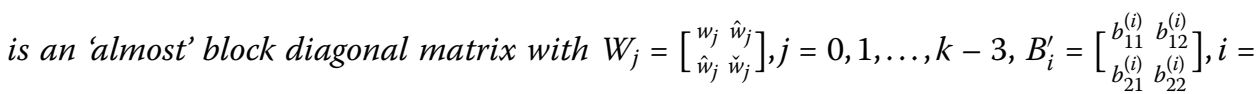
$1,2,3,4$ satisfying $w_{i}, \hat{w}_{i}, \check{w}_{i}>0, \operatorname{det}\left(W_{i}\right)>0, i=0,1, \ldots, k-3, \frac{w_{i} \hat{w}_{i+1}-\hat{w}_{i} w_{i+1}}{w_{i+1} \operatorname{det}\left(W_{i}\right)}=\frac{d_{i+2}}{\check{d}_{i+2}}, i=$ $0,1, \ldots, k-4$, where $\dot{d}_{i+2}, \grave{d}_{i+2}, i=0,1, \ldots, k-4$ are entries of $\mathbf{J}_{b}$ as mentioned in Lemma 3.1, 
and $\operatorname{det}\left(B_{i}^{\prime}\right) \neq 0, i=1,2,3,4$. Then there exists a block pseudo-Jacobi matrix $\mathbf{M} \in \mathbb{M}_{2 k}$ such that

$$
\begin{aligned}
& \sigma(\mathbf{M}, \mathbf{W})=\left\{\lambda_{i}: i=1, \ldots, 2 k\right\}, \\
& \sigma\left(\mathbf{M}_{12}\left[-E_{2}\left(B_{1}^{\prime}\right)^{-1} B_{2}^{\prime}\right], \mathbf{W}_{12}\right)=\left\{\xi_{i}: i=1, \ldots, 2 k-2\right\} .
\end{aligned}
$$

Proof Since $\left\{\lambda_{i}: i=1, \ldots, 2 k\right\},\left\{\mu_{i}: i=1, \ldots, 2 k-1\right\}$ and $\left\{\xi_{i}: i=1, \ldots, 2 k-2\right\}$ are three sets of real numbers satisfying the interlacing property (3.2), hence by Lemma 3.1, there exists a two-banded matrix $\mathbf{J}_{b} \in \mathbb{M}_{2 k}$ such that

$$
\begin{aligned}
& \sigma\left(\mathbf{J}_{b}\right)=\left\{\lambda_{i}: i=1, \ldots, k\right\}, \quad \sigma\left(\left(\mathbf{J}_{b}\right)_{1}\right)=\left\{\mu_{i}: i=1, \ldots, k-1\right\}, \\
& \sigma\left(\left(\mathbf{J}_{b}\right)_{12}\right)=\left\{\xi_{i}: i=1, \ldots, k-2\right\} .
\end{aligned}
$$

Now for each $\lambda=\lambda_{i}, i=1, \ldots, 2 k$, there exists a nontrivial $u \in \mathbb{R}^{2 k}$ such that

$$
\left(\mathbf{J}_{b}-\lambda I_{2 k}\right) u=0 .
$$

Set

$$
\mathbf{H}=\left[\begin{array}{ccccc}
I_{2} & -\left(B_{1}^{\prime}\right)^{-1} B_{2}^{\prime} & & & \\
& I_{2} & & & \\
& & \cdots & & \\
& & & I_{2} & \\
& & & -\left(B_{4}^{\prime}\right)^{-1} B_{3}^{\prime} & I_{2}
\end{array}\right],
$$

then one has $\mathbf{W H}=\mathbf{L} \mathbf{U}$, with $\mathbf{L}=\operatorname{diag}\left(L_{1}, R_{0}^{T}, R_{1}^{T}, \ldots, R_{k-3}^{T}, L_{2}\right), \mathbf{U}=\operatorname{diag}\left(U_{1}, R_{0}, R_{1}, \ldots\right.$, $\left.R_{k-3}, U_{2}\right)$, where $U_{1}=\left(R_{0}^{T}\right)^{-1}\left(\mathcal{D}_{1}^{T}\right)^{-1} E_{2}, U_{2}=\left(R_{k-3}^{T}\right)^{-1}\left(\mathcal{D}_{k-1}\right)^{-1}\left(-E_{2}\right), L_{1}=-B_{1}^{\prime}\left(U_{1}\right)^{-1}, L_{2}=$ $-B_{4}^{\prime}\left(U_{2}\right)^{-1}, R_{i}=\left[\begin{array}{cc}\sqrt{w_{i}} & \frac{\hat{w}_{i}}{\sqrt{w_{i}}} \\ 0 & \frac{\sqrt{\hat{w}_{i} w_{i}-\hat{w}_{i}^{2}}}{\sqrt{w_{i}}}\end{array}\right], i=0,1, \ldots, k-3, \mathcal{D}_{1}^{T}, \mathcal{D}_{k-1} \in \mathbf{J}_{b}$.

Let $u=\mathbf{U H}^{-1} \tilde{u}$ and left multiplying Eq. (3.5) by $\mathbf{L}$ we obtain

$$
\left(\mathbf{L} \mathbf{J}_{b} \mathbf{U H}^{-1}-\lambda \mathbf{L} \mathbf{U H}^{-1}\right) \tilde{u}=0, \quad \text { i.e., } \quad(\mathbf{M}-\lambda \mathbf{W}) \tilde{u}=0,
$$

where $\mathbf{M}=\mathbf{L}_{b} \mathbf{U H}^{-1}$. Clearly, $\lambda \in \sigma(\mathbf{M}, \mathbf{W})$ and $\mathbf{M} \in \mathbb{M}_{2 k}$ is a block pseudo-Jacobi matrix. The same argument applies to each $\xi=\xi_{i}, i=1, \ldots, 2 k-2$, then we obtain the conclusion that $\mathbf{M}_{12}\left[-E_{2}\left(B_{1}^{\prime}\right)^{-1} B_{2}^{\prime}\right]=\mathbf{L}_{12}\left(\mathbf{J}_{b}\right)_{12} \mathbf{U}_{12}\left(\mathbf{H}^{-1}\right)_{12}, \mathbf{W}_{12}=\mathbf{L}_{12} \mathbf{U}_{12}\left(\mathbf{H}^{-1}\right)_{12}$ and $\xi \in \sigma\left(\mathbf{M}_{12}, \mathbf{W}_{12}\right)$. Thus,

$$
\sigma\left(\mathbf{J}_{b}\right) \subset \sigma(\mathbf{M}, \mathbf{W}) \text { and } \sigma\left(\left(\mathbf{J}_{b}\right)_{12}\right) \subset \sigma\left(\mathbf{M}_{12}\left[-E_{2}\left(B_{1}^{\prime}\right)^{-1} B_{2}^{\prime}\right], \mathbf{W}_{12}\right) .
$$

Similarly by reversing the steps above we can get

$$
\sigma\left(\mathbf{J}_{b}\right) \supset \sigma(\mathbf{M}, \mathbf{W}) \text { and } \sigma\left(\left(\mathbf{J}_{b}\right)_{12}\right) \supset \sigma\left(\mathbf{M}_{12}\left[-E_{2}\left(B_{1}^{\prime}\right)^{-1} B_{2}^{\prime}\right], \mathbf{W}_{12}\right) \text {. }
$$

Consequently,

$$
\sigma\left(\mathbf{J}_{b}\right)=\sigma(\mathbf{M}, \mathbf{W}) \quad \text { and } \quad \sigma\left(\left(\mathbf{J}_{b}\right)_{12}\right)=\sigma\left(\mathbf{M}_{12}\left[-E_{2}\left(B_{1}^{\prime}\right)^{-1} B_{2}^{\prime}\right], \mathbf{W}_{12}\right) .
$$

This completes the proof. 
Corollary 3.1 When $\mathbf{M}_{12}\left[-E_{2}\left(B_{1}^{\prime}\right)^{-1} B_{2}^{\prime}\right]$ and $\mathbf{W}_{12}$ are replaced by $\mathbf{M}^{12}\left[E_{2}\left(B_{4}^{\prime}\right)^{-1} B_{3}^{\prime}\right]$ and $\mathbf{W}^{12}$, respectively, then the similar conclusion of Theorem 3.1 still holds.

Corollary 3.2 When $w_{i}, \hat{w}_{i}, \check{w}_{i}>0$ are replaced by $w_{i}, \hat{w}_{i}, \check{w}_{i}<0$ for $i=1, \ldots, k$, then the similar conclusion of Theorem 3.1 still holds.

\section{Main result}

We now state our main result on the inverse spectral problem of the fourth-order eigenparameter-dependent BVPs (1.1), (1.2).

Theorem 4.1 Let $\left\{\lambda_{i}: i=1, \ldots, 2 k\right\},\left\{\mu_{i}: i=1, \ldots, 2 k-1\right\}$ and $\left\{\xi_{i}: i=1, \ldots, 2 k-2\right\}$ be three sets of real numbers satisfying the interlacing property (3.2). Let $n=k-3$. Then, for any $-\infty<a<b<+\infty$, any partition (1.4), any $w \in L(I, \mathbb{R})$ satisfying (1.7), any real numbers $\alpha_{i}^{\prime}, \beta_{i}^{\prime}, \gamma_{i}^{\prime}, \delta_{i}^{\prime}, i=1,2,3,4$ satisfying $\alpha_{1}^{\prime} \beta_{2}^{\prime}-\alpha_{2}^{\prime} \beta_{1}^{\prime} \neq 0, \alpha_{3}^{\prime} \beta_{4}^{\prime}-\alpha_{4}^{\prime} \beta_{3}^{\prime} \neq 0, \gamma_{1}^{\prime} \delta_{2}^{\prime}-\gamma_{2}^{\prime} \delta_{1}^{\prime} \neq 0, \gamma_{3}^{\prime} \delta_{4}^{\prime}-$ $\gamma_{4}^{\prime} \delta_{3}^{\prime} \neq 0$, we have the following:

(a) There exist $r, q \in L(I, \mathbb{R})$ satisfying (1.5) and (1.6), the values of eigenparameterdependent $B C s \alpha_{i}, \beta_{i}, \gamma_{i}, \delta_{i}, i=1,2,3,4$, such that the associated equivalent family of the fourth-order eigenparameter-dependent BVPs (1.1), (1.2) has the spectrum

$$
\begin{aligned}
& \sigma\left(\vec{\alpha}, \overrightarrow{\alpha^{\prime}}, \vec{\beta}, \vec{\beta}^{\prime}, \vec{\gamma}, \vec{\gamma}^{\prime}, \vec{\delta}, \vec{\delta}^{\prime}\right)=\left\{\lambda_{i}: i=1, \ldots, 2 k\right\}, \\
& \sigma\left(\overrightarrow{0}, \overrightarrow{\alpha^{\prime}}, \overrightarrow{0}, \overrightarrow{\beta^{\prime}}, \vec{\gamma}, \overrightarrow{\gamma^{\prime}}, \vec{\delta}, \tilde{\delta}^{\prime}\right)=\left\{\xi_{i}: i=1, \ldots, 2 k-2\right\} .
\end{aligned}
$$

(b) There exist $r, q \in L(I, \mathbb{R})$ satisfying (1.5) and (1.6), the values of eigenparameterdependent $B C s \alpha_{i}, \beta_{i}, \gamma_{i}, \delta_{i}, i=1,2,3,4$, such that the associated equivalent family of the fourth-order eigenparameter-dependent BVPs (1.1), (1.2) has the spectrum

$$
\begin{aligned}
& \sigma\left(\vec{\alpha}, \overrightarrow{\alpha^{\prime}}, \vec{\beta}, \vec{\beta}^{\prime}, \vec{\gamma}, \overrightarrow{\gamma^{\prime}}, \vec{\delta}, \vec{\delta}^{\prime}\right)=\left\{\lambda_{i}: i=1, \ldots, 2 k\right\}, \\
& \sigma\left(\vec{\alpha}, \vec{\alpha}^{\prime}, \vec{\beta}, \vec{\beta}^{\prime}, \overrightarrow{0}, \vec{\gamma}^{\prime}, \overrightarrow{0}, \vec{\delta}^{\prime}\right)=\left\{\xi_{i}: i=1, \ldots, 2 k-2\right\} .
\end{aligned}
$$

Proof (a) For any $-\infty<a<b<+\infty$ and a given partition (1.4) of [a,b], define $w_{i}=$ $\int_{a_{i}}^{b_{i}} w(t) d t, \hat{w}_{i}=\int_{a_{i}}^{b_{i}} w(t) t d t, \check{w}_{i}=\int_{a_{i}}^{b_{i}} w(t) t^{2} d t, i=0,1, \ldots, n, W_{i}=\left[\begin{array}{c}w_{i} \\ \hat{w}_{i} \\ \hat{w}_{i}\end{array}\right], i=0,1, \ldots, n$, satisfying the conditions $\frac{w_{i} \hat{w}_{i+1}-\hat{w}_{i} w_{i+1}}{w_{i+1} \operatorname{det}\left(W_{i}\right)}=\frac{\dot{d}_{i+2}}{d_{i+2}}, i=0,1, \ldots, n-1$. For any real numbers $\alpha_{i}^{\prime}, \beta_{i}^{\prime}, \gamma_{i}^{\prime}, \delta_{i}^{\prime}$, $i=1,2,3,4$, satisfying $\alpha_{1}^{\prime} \beta_{2}^{\prime}-\alpha_{2}^{\prime} \beta_{1}^{\prime} \neq 0, \alpha_{3}^{\prime} \beta_{4}^{\prime}-\alpha_{4}^{\prime} \beta_{3}^{\prime} \neq 0, \gamma_{1}^{\prime} \delta_{2}^{\prime}-\gamma_{2}^{\prime} \delta_{1}^{\prime} \neq 0, \gamma_{3}^{\prime} \delta_{4}^{\prime}-\gamma_{4}^{\prime} \delta_{3}^{\prime} \neq 0$, define $B_{1}^{\prime}=\left[\begin{array}{c}\alpha_{3}^{\prime} a \alpha_{3}^{\prime}+\alpha_{4}^{\prime} \\ \beta_{3}^{\prime} a \beta_{3}^{\prime}+\beta_{4}^{\prime}\end{array}\right], B_{2}^{\prime}=\left[\begin{array}{c}\alpha_{1}^{\prime} a \alpha_{1}^{\prime}+\alpha_{2}^{\prime} \\ \beta_{1}^{\prime} a \beta_{1}^{\prime}+\beta_{2}^{\prime}\end{array}\right], B_{3}^{\prime}=\left[\begin{array}{l}\gamma_{3}^{\prime} b \gamma_{3}^{\prime}+\gamma_{4}^{\prime} \\ \delta_{3}^{\prime} b \delta_{3}^{\prime}+\delta_{4}^{\prime}\end{array}\right], B_{4}^{\prime}=\left[\begin{array}{c}\gamma_{1}^{\prime} b \gamma_{1}^{\prime}+\gamma_{2}^{\prime} \\ \delta_{1}^{\prime} b \delta_{1}^{\prime}+\delta_{2}^{\prime}\end{array}\right]$. Then define

$$
\mathbf{W}=\left[\begin{array}{ccccccc}
-B_{1}^{\prime} & -B_{2}^{\prime} & & & & & \\
& W_{0} & & & & & \\
& & W_{1} & & & & \\
& & & \ldots & & & \\
& & & & W_{n-1} & & \\
& & & & & W_{n} & \\
& & & & & -B_{3}^{\prime} & -B_{4}^{\prime}
\end{array}\right] .
$$

By (1.7), $w_{i}>0, \hat{w}_{i}>0, \check{w}_{i}>0, i=0,1, \ldots, n$, and $\operatorname{det}\left(B_{i}^{\prime}\right) \neq 0, i=1,2,3,4$. Since $k=n+3$, by Theorem 3.1, there exists a block pseudo-Jacobi matrix $\mathbf{M} \in \mathbb{M}_{2 n+6}$ in the form of (3.3) 
satisfying $D_{1}^{\prime}=-D_{k-1}^{\prime}=E_{2}$ such that

$$
\begin{aligned}
& \sigma(\mathbf{M}, \mathbf{W})=\left\{\lambda_{i}: i=1, \ldots, 2 n+6\right\}, \\
& \sigma\left(\mathbf{M}_{12}\left[-E_{2}\left(B_{1}^{\prime}\right)^{-1} B_{2}^{\prime}\right], \mathbf{W}_{12}\right)=\left\{\xi_{i}: i=1, \ldots, 2 n+4\right\} .
\end{aligned}
$$

Let

$$
\begin{aligned}
& \theta_{i}=\frac{1}{\hat{d}_{i}^{2}-\check{d}_{i} d_{i}}, \quad i=1, \ldots, n ; \\
& r_{i}=\theta_{i} d_{i}, \quad \hat{r}_{i}=\theta_{i} \hat{d}_{i}, \quad \check{r}_{i}=\theta_{i} \check{d}_{i}, \quad i=1, \ldots, n ; \\
& q_{i}=c_{i+2}+\frac{r_{i}}{\theta_{i}}+\frac{r_{i+1}}{\theta_{i+1}}, \quad \hat{q}_{i}=\hat{c}_{i+2}+\frac{\hat{r}_{i}}{\theta_{i}}+\frac{\hat{r}_{i+1}}{\theta_{i+1}}, \\
& \check{q}_{i}=\check{c}_{i+2}+\frac{\check{r}_{i}}{\theta_{i}}+\frac{\check{r}_{i+1}}{\theta_{i+1}}, \quad i=1, \ldots, n-1, \\
& q_{0}=c_{2}+\frac{r_{1}}{\theta_{1}}, \quad \hat{q}_{0}=\hat{c}_{2}+\frac{\hat{r}_{1}}{\theta_{1}}, \quad \check{q}_{0}=\check{c}_{2}+\frac{\check{r}_{1}}{\theta_{1}}, \\
& q_{n}=c_{n+2}+\frac{r_{n}}{\theta_{n}}, \quad \hat{q}_{n}=\hat{c}_{n+2}+\frac{\hat{r}_{n}}{\theta_{n}}, \quad \check{q}_{n}=\check{c}_{n+2}+\frac{\check{r}_{n}}{\theta_{n}} ; \\
& \alpha_{1}=d_{11}^{(1)}, \quad \alpha_{2}=d_{12}^{(1)}-a * d_{11}^{(1)}, \quad \beta_{1}=d_{21}^{(1)}, \quad \beta_{2}=d_{22}^{(1)}-a * d_{21}^{(1)}, \\
& \alpha_{3}=c_{11}^{(1)}, \quad \alpha_{4}=c_{12}^{(1)}-a * c_{11}^{(1)}, \quad \beta_{3}=c_{21}^{(1)}, \quad \beta_{4}=c_{22}^{(1)}-a * c_{21}^{(1)}, \\
& \gamma_{1}=d_{11}^{(k-1)}, \quad \gamma_{2}=d_{12}^{(k-1)}-b * d_{11}^{(k-1)}, \quad \delta_{1}=d_{21}^{(k-1)}, \quad \delta_{2}=d_{22}^{(k-1)}-b * d_{21}^{(k-1)}, \\
& \gamma_{3}=c_{11}^{(k)}, \quad \gamma_{4}=c_{12}^{(k)}-b * c_{11}^{(k)}, \quad \delta_{3}=c_{21}^{(k)}, \quad \delta_{4}=c_{22}^{(k)}-b * c_{21}^{(k)},
\end{aligned}
$$

and define $\mathbf{P}, \mathbf{Q}, \mathbf{P}_{12}\left[-E_{2}\left(B_{1}^{\prime}\right)^{-1} B_{2}^{\prime}\right]$ and $\mathbf{Q}_{12}$ by (2.3), (2.4), (2.6) and (2.7), respectively. Clearly, $r_{i}, \hat{r}_{i}, \check{r}_{i} \neq 0, i=1, \ldots, n$. It is easy to see that $\mathbf{M}=\mathbf{P}+\mathbf{Q}$ and $\mathbf{M}_{12}\left[-E_{2}\left(B_{1}^{\prime}\right)^{-1} B_{2}^{\prime}\right]=$ $\mathbf{P}_{12}\left[-E_{2}\left(B_{1}^{\prime}\right)^{-1} B_{2}^{\prime}\right]+\mathbf{Q}_{12}$. With the notation in (2.5) and (2.8), we also have $(\mathbf{W})_{12}=\mathbf{W}_{12}$. Therefore,

$$
\begin{aligned}
& \sigma(\mathbf{P}+\mathbf{Q}, \mathbf{W})=\left\{\lambda_{i}: i=1, \ldots, 2 n+6\right\}, \\
& \sigma\left(\mathbf{P}_{12}\left[-E_{2}\left(B_{1}^{\prime}\right)^{-1} B_{2}^{\prime}\right]+\mathbf{Q}_{12}, \mathbf{W}_{12}\right)=\left\{\xi_{i}: i=1, \ldots, 2 n+4\right\} .
\end{aligned}
$$

By Lemmas 2.3 and 2.4 we see that for the fourth-order eigenparameter-dependent BVPs

$$
\begin{aligned}
& \sigma\left(\vec{\alpha}, \overrightarrow{\alpha^{\prime}}, \vec{\beta}, \overrightarrow{\beta^{\prime}}, \vec{\gamma}, \overrightarrow{\gamma^{\prime}}, \vec{\delta}, \vec{\delta}^{\prime}\right)=\left\{\lambda_{i}: i=1, \ldots, 2 n+6\right\}, \\
& \sigma\left(\overrightarrow{0}, \overrightarrow{\alpha^{\prime}}, \overrightarrow{0}, \vec{\beta}^{\prime}, \vec{\gamma}, \overrightarrow{\gamma^{\prime}}, \vec{\delta}, \tilde{\delta^{\prime}}\right)=\left\{\xi_{i}: i=1, \ldots, 2 n+4\right\} .
\end{aligned}
$$

We observe that the choice of $r_{i}, \hat{r}_{i}, \breve{r}_{i}, i=1, \ldots, n$ and $q_{i}, \hat{q}_{i}, \breve{q}_{i}, i=0, \ldots, n$ and all $r, q \in L(I, \mathbb{R})$ by this choice form an equivalent family of the fourth-order eigenparameter-dependent BVPs. This completes the proof.

(b) The proof is similar using Corollary 3.1, and Lemmas 2.3, 2.5, hence the details are omitted here.

To illustrate our main result we show the following algorithm and example. 


\section{Algorithm 1}

Step 1. Input three sets of real numbers $\lambda_{1}, \lambda_{2}, \ldots, \lambda_{2 k}, \mu_{1}, \mu_{2}, \ldots, \mu_{2 k-1}$ and $\xi_{1}, \xi_{2}, \ldots, \xi_{2 k-2}$.

Step 2. Construct the matrix $\mathbf{J}_{b}$ by the proof of Problem 2.6 in [22].

Step 3. Set $a, b$ and the partition of $[a, b]$. Input $\bar{w}(t)$ and $\alpha_{i}^{\prime}, \beta_{i}^{\prime}, \gamma_{i}^{\prime}, \delta_{i}^{\prime} \in \mathbb{R}, i=1,2,3,4$. Set $\mathbf{W}$ and $\mathbf{H}$ to obtain $\mathbf{L}$ and $\mathbf{U}$. Compute $\mathbf{M}=\mathbf{L} \mathbf{J}_{b} \mathbf{U H}^{-1}$.

Step 4. Obtain $\alpha_{i}, \beta_{i}, \gamma_{i}, \delta_{i} \in \mathbb{R}, i=1,2,3,4$, and $r_{i}, \hat{r}_{i}, \breve{r}_{i}, i=1, \ldots, n, q_{i}, \hat{q}_{i}, \check{q}_{i}, i=0, \ldots, n$ by Theorem 4.1.

Example 1 Given three sets of real numbers satisfying the interlacing property (3.2): $\lambda_{1}=$ $-3.8397, \lambda_{2}=-3.0377, \lambda_{3}=-0.0540, \lambda_{4}=2.5830, \lambda_{5}=5.5791, \lambda_{6}=7.0055, \lambda_{7}=11.3818$, $\lambda_{8}=15.3820 ; \mu_{1}=-3.0842, \mu_{2}=-1.9058, \mu_{3}=2.3327, \mu_{4}=3.2462, \mu_{5}=6.2319, \mu_{6}=$ 9.4212, $\mu_{7}=13.7579 ; \xi_{1}=-2.7411, \xi_{2}=-1.3502, \xi_{3}=3.1316, \xi_{4}=5.7604, \xi_{5}=8.0532, \xi_{6}=$ 13.1461. Let $\alpha_{1}^{\prime}=-10, \alpha_{2}^{\prime}=-12, \alpha_{3}^{\prime}=-5, \alpha_{4}^{\prime}=-14, \beta_{1}^{\prime}=-4, \beta_{2}^{\prime}=-2, \beta_{3}^{\prime}=-2, \beta_{4}^{\prime}=-7, \gamma_{1}^{\prime}=$ $9, \gamma_{2}^{\prime}=-33, \gamma_{3}^{\prime}=-3, \gamma_{4}^{\prime}=7, \delta_{1}^{\prime}=3, \delta_{2}^{\prime}=-15, \delta_{3}^{\prime}=-1, \delta_{4}^{\prime}=1, n=1$. Given an interval $I=$ $[-2,3]$ and a partition of it: $-2<0<1<3$. Define the piecewise polynomial function $\bar{w}$ on $I$ by

$$
\bar{w}(t)= \begin{cases}0.7624 t^{2}-0.1236 t-0.1401, & t \in[-2,0), \\ 0, & t \in[0,1), \\ -3.2811 t^{2}+12.3742 t-9.0306, & t \in[1,3] .\end{cases}
$$

Then we can obtain the fourth-order eigenparameter-dependent boundary value problem:

$$
\left\{\begin{array}{l}
\left(\bar{p} y^{\prime \prime}\right)^{\prime \prime}+\bar{q} y=\lambda \bar{w} y, \quad \text { on } I=[-2,3], \\
(-10 \lambda+483.2857) y(-2)+(-12 \lambda+1587.3571) y^{\prime}(-2) \\
\quad+(-5 \lambda+36.1429)\left(p y^{\prime}\right)(-2)+(-14 \lambda+104.1429)\left(p y^{\prime \prime}\right)^{\prime}(-2)=0, \\
(-4 \lambda+29.7143) y(-2)+(-2 \lambda+81.1429) y^{\prime}(-2) \\
\quad+(-2 \lambda+6.8571)\left(p y^{\prime}\right)(-2)+(-7 \lambda+22.8571)\left(p y^{\prime \prime}\right)^{\prime}(-2)=0, \\
(9 \lambda-522.0000) y(3)+(-33 \lambda+1380.9000) y^{\prime}(3) \\
\quad+(-3 \lambda+76.5000)\left(p y^{\prime \prime}\right)(3)+(7 \lambda-96.4500)\left(p y^{\prime \prime}\right)^{\prime}(3)=0, \\
(3 \lambda-14.0000) y(3)+(-15 \lambda+74.9667) y^{\prime}(3) \\
\quad+(-\lambda+5.5000)\left(p y^{\prime \prime}\right)(3)+(\lambda-6.1500)\left(p y^{\prime \prime}\right)^{\prime}(3)=0,
\end{array}\right.
$$

where the piecewise polynomial functions $\bar{r}=\frac{1}{\bar{p}}$ and $\bar{q}$ on $I=[-2,3]$ are as follows:

$$
\begin{aligned}
& \bar{r}(t)= \begin{cases}0, & t \in[-2,0), \\
211.2683 t^{2}-201.4703 t+30.9248, & t \in[0,1), \\
0, & t \in[1,3] .\end{cases} \\
& \bar{q}(t)= \begin{cases}8.5514 t^{2}-9.2826 t-12.0102, & t \in[-2,0), \\
0, & t \in[0,1), \\
-80.7883 t^{2}+325.0663 t-291.8755, & t \in[1,3] .\end{cases}
\end{aligned}
$$


Hence the spectrum of the reconstructed fourth-order eigenparameter-dependent BVPs (1.1), (1.2) is

$$
\left\{\begin{aligned}
\sigma & \left(\vec{\alpha}, \overrightarrow{\alpha^{\prime}}, \vec{\beta}, \vec{\beta}^{\prime}, \vec{\gamma}, \overrightarrow{\gamma^{\prime}}, \vec{\delta}, \vec{\delta}^{\prime}\right) \\
& =\{-3.8397,-3.0377,-0.0540,2.5830,5.5791,7.0055,11.3818,15.3820\} \\
\sigma & \left(0, \vec{\alpha}^{\prime}, 0, \overrightarrow{\beta^{\prime}}, \vec{\gamma}, \overrightarrow{\gamma^{\prime}}, \vec{\delta}, \vec{\delta}^{\prime}\right) \\
& =\{-2.7411,-1.3502,3.1316,5.7604,8.0532,13.1461\} .
\end{aligned}\right.
$$

\section{Acknowledgements}

The authors sincerely thank the referees for their helpful comments and detailed suggestions. These have significantly improved the presentation of this paper.

\section{Funding}

This work was supported by National Natural Science Foundation of China (Grant Nos. 11661059, 11301259), Natural Science Foundation of Inner Mongolia (Grant No. 2017JQ07).

\section{Availability of data and materials}

No data were used to support this study.

\section{Conflicts of interest}

The authors declare that they have no conflicts of interest.

\section{Competing interests}

The authors declare that they have no competing interests.

\section{Authors' contributions}

The authors equally contributed this work. All authors read and approved the final manuscript.

\section{Publisher's Note}

Springer Nature remains neutral with regard to jurisdictional claims in published maps and institutional affiliations.

Received: 26 September 2019 Accepted: 15 January 2020 Published online: 21 January 2020

\section{References}

1. Ao, J.J., Bo, F.Z:: Matrix representations of fourth order boundary value problems with eigenparameter-dependent boundary conditions. Acta Math. Sinica (Chin. Ser.) 60, 427-438 (2017) (in Chinese)

2. Ao, J.J., Sun, J., Zettl, A.: Finite spectrum of 2nth order boundary value problems. Appl. Math. Lett. 42, 1-8 (2015)

3. Ao, J.J., Sun, J., Zhang, M.Z.: The finite spectrum of Sturm-Liouville problems with transmission conditions. Appl. Math. Comput. 218, 1166-1173 (2011)

4. Ao, J.J., Sun, J., Zhang, M.Z.: Equivalence of fourth order boundary value problems and matrix eigenvalue problems. Results Math. 63, 581-595 (2013)

5. Ao, J.J., Zhang, L.: Inverse spectral problem of fourth-order boundary value problems with finite spectrum. Math. Methods Appl. Sci. 42, 4472-4479 (2019)

6. Atkinson, F.V.: Discrete and Continuous Boundary Problems. Academic Press, New York (1964)

7. Bebinao, N., Providência, J.D.: Inverse problems for pseudo-Jacobi matrices: existence and uniqueness results. Inverse Probl. 27, 025005 (2011)

8. Borg, G.: Eine Umkehrung der Sturm-Liouvilleschen Eigenwertaufgabe. Acta Math. 78, 1-96 (1946)

9. Cai, J.M., Zheng, Z.W.: Inverse spectral problems for discontinuous Sturm-Liouville problems of Atkinson type. Appl. Math. Comput. 327, 22-34 (2018)

10. Freiling, G., Yurko, V.A.: Inverse Sturm-Liouville Problems and Their Applications. NOVA Science Publishers, New York (2001)

11. Fu, S.Z., Xu, Z.B., Wei, G.S.: The interlacing of spectra between continuous and discontinuous Sturm-Liouville problems and its application to inverse problems. Taiwan. J. Math. 16, 651-663 (2012)

12. Gladwell, M.: Inverse Problems in Vibrations. Kluwer Academic, Dordrecht (2004)

13. Hochstadt, H.: On some inverse problems in matrix theory. Arch. Math. 18, $201-207$ (1967)

14. Kong, Q., Volkmer, H., Zettl, A.: Matrix representations of Sturm-Liouville problems with finite spectrum. Results Math. $54,103-116(2009)$

15. Kong, Q., Wu, H., Zettl, A.: Sturm-Liouville problems with finite spectrum. J. Math. Anal. Appl. 263, 748-762 (2001)

16. Kong, Q., Zettl, A.: Inverse Sturm-Liouville problems with finite spectrum. J. Math. Anal. Appl. 386, 1-9 (2012)

17. Mirzaei, H., Ghanbari, K.: Matrix representation of a sixth order Sturm-Liouville problem and related inverse problem with finite spectrum. Bull. Iran. Math. Soc. 41, 1031-1043 (2015)

18. Shieh, C.T.: Some inverse problems on Jacobi matrices. Inverse Probl. 20, 589-600 (2004)

19. Simon, B.: The classical moment problem as a self-adjoint finite difference operator. Adv. Math. 137, 82-203 (1998)

20. Tretter, $C$ : Boundary eigenvalue problems with differential equations $N \eta=\lambda P \eta$ with $\lambda$-polynomial boundary conditions. J. Differ. Equ. 170, 408-471 (2001) 
21. Volkmer, H.: Eigenvalue problems of Atkinson, Feller and Krein and their mutual relationship. Electron. J. Differ. Equ. 2005, 48 (2005)

22. Xu, S.F: An Introduction to Inverse Algebraic Eigenvalue Problems. Peking University Press, Beijing (1998)

23. Yang, C.F., Yang, X.P.: An interior inverse problem for the Sturm-Liouville operator with discontinuous conditions. Appl. Math. Lett. 22, 1315-1319 (2009)

24. Yurko, V.A.: An inverse problem for higher order differential operators on star-type graphs. Inverse Probl. 23, 893-903 (2007)

25. Yurko, V.A.: Inverse problems for second order integro-differential operators. Appl. Math. Lett. 74, 1-6 (2017)

26. Zhang, L., Ao, J.J.: On a class of inverse Sturm-Liouville problems with eigenparameter dependent boundary conditions. Appl. Math. Comput. 2019, 362 (2019). https://doi.org/10.1016/j.amc.2019.06.067

27. Zhang, L., Ao, J.J.: Inverse spectral problem for Sturm-Liouville operator with coupled eigenparameter dependent boundary conditions of Atkinson type. Inverse Probl. Sci. Eng. 27, 1689-1702 (2019)

\section{Submit your manuscript to a SpringerOpen ${ }^{\circ}$} journal and benefit from:

- Convenient online submission

$\checkmark$ Rigorous peer review

- Open access: articles freely available online

- High visibility within the field

Retaining the copyright to your article

Submit your next manuscript at $\gg$ springeropen.com 